\title{
TROPICAL HODGE NUMBERS OF NON-ARCHIMEDEAN CURVES
}

\author{
PHILIPP JELL
}

\begin{abstract}
We study the tropical Dolbeault cohomology of non-archimedean curves as defined by Chambert-Loir and Ducros. We give a precise condition for when this cohomology satisfies Poincaré duality. The condition is always satisfied when the residue field of the non-archimedean base field is the algebraic closure of a finite field. We also show that for curves over fields with residue field $\mathbb{C}$, the tropical $(1,1)$-Dolbeault cohomology can be infinite dimensional. Our main new ingredient is an exponential type sequence that relates tropical Dolbeault cohomology to the cohomology of the sheaf of harmonic functions. As an application of our Poincaré duality result, we calculate the dimensions of the tropical Dolbeault cohomology, called tropical Hodge numbers, for (open subsets of) curves.
\end{abstract}

MSC: Primary 14G22; Secondary 14G40, 32P05

\section{INTRODUCTION}

Let $K$ be a field that is complete with respect to a non-archimedean absolute value. For a $K$-analytic space $X$ in the sense of V. Berkovich [Ber90] A. Chambert-Loir and A. Ducros introduced a double complex $\left(\mathcal{A}^{\bullet, \bullet}, d^{\prime}, d^{\prime \prime}\right)$ of sheaves of smooth real-valued differential forms on $X$ [CLD12]. These bigraded differential forms are analogues of smooth differential forms on complex manifolds. We consider these forms when the analytic space $X^{\text {an }}$ is the associated analytic space of a variety $X$ over $K$. We always assume that $K$ is algebraically closed and that its absolute value is non-trivial.

A natural question that arose in this context concerns the behavior of the associated Dolbeault cohomology

$$
\mathrm{H}^{p, q}\left(X^{\mathrm{an}}\right):=\frac{\operatorname{ker}\left(d^{\prime \prime}: \mathcal{A}^{p, q}\left(X^{\mathrm{an}}\right) \rightarrow \mathcal{A}^{p, q+1}\left(X^{\mathrm{an}}\right)\right)}{\operatorname{im}\left(d^{\prime \prime}: \mathcal{A}^{p, q-1}\left(X^{\mathrm{an}}\right) \rightarrow \mathcal{A}^{p, q}\left(X^{\mathrm{an}}\right)\right)} .
$$

and the associated tropical Hodge numbers $h^{p, q}\left(X^{\text {an }}\right):=\operatorname{dim}_{\mathbb{R}} \mathrm{H}^{p, q}\left(X^{\text {an }}\right)$. Concretely, one may ask whether, for smooth proper varieties $X$ of dimension $n$, this cohomology satisfies a version of Poincaré duality, i.e. if the canonical pairing $\mathrm{H}^{p, q}\left(X^{\text {an }}\right) \times$ $\mathrm{H}^{n-p, n-q}\left(X^{\text {an }}\right) \rightarrow \mathbb{R}$ is a perfect pairing for all $p$ and $q$. This implies the symmetry $h^{p, q}\left(X^{\text {an }}\right)=h^{n-p, n-q}\left(X^{\text {an }}\right)$ for the tropical Hodge numbers. We give a precise condition when Poincaré duality holds for curves.

Theorem A (Theorem 4.2, Remark 3.6). Let $X$ be a smooth projective curve over $K$. Then $X$ satisfies Poincaré duality if and only if there exists a strictly semistable model of $X$ over the valuation ring of $K$ such that for every irreducible component $C$ of the special fiber the abelian group $\operatorname{Pic}^{0}(C)$ is torsion.

Let us state some consequences and remarks.

The author was supported by the collaborative research center CRC 1085 "Higher Invariants" funded by the Deutsche Forschungsgemeinschaft. 
- Theorem A implies that all smooth projective curves over $K$ satisfy Poincaré duality if the residue field of $K$ is the algebraic closure of a finite field (see Corollary 4.3).

- Theorem A enables us to calculate the tropical Hodge numbers $h^{p, q}\left(X^{\text {an }}\right)$ (see Theorem 4.8).

- Using Theorem A we are able to establish the case of curves for a conjecture by Y. Liu [Liu17a, Conjecture 5.2] (see Theorem 4.9).

- The property that $\operatorname{Pic}^{0}(C)$ is torsion for all irreducible components of the special fiber holds for all strictly semistable models of $X$ if it holds for one strictly semistable model of $X$ (see Remark 3.6).

We also show that in general $\mathrm{H}^{1,1}\left(X^{\text {an }}\right)$ is not finite dimensional.

Theorem B (Theorem 4.4, Remark 3.6). Let $X$ be a smooth projective curve over $K$ and let the residue field of $K$ be $\mathbb{C}$. Then $\mathrm{H}^{1,1}(X)$ is finite dimensional if and only if there exists a strictly semistable model of $X$ such that the special fiber has only rational irreducible components.

Again, if the property that all irreducible components of the special fiber are rational holds for one strictly semistable model, it holds for all strictly semistable models (see Remark 3.6). In particular for $K$ as in Theorem B, the real vector space $\mathrm{H}^{1,1}\left(X^{\text {an }}\right)$ is infinite dimensional for all smooth projective curves $X$ of positive genus and good reduction.

1.1. Relation with previous results. Now let $K$ again be any algebraically closed complete non-archimedean field and let $X$ be a variety over $K$. The author showed in [Jel16b, Corollary 4.6] that for all $q$ the cohomology $\mathrm{H}^{0, q}\left(X^{\text {an }}\right)$ agrees with $\mathrm{H}_{\text {sing }}^{q}\left(X^{\text {an }}, \mathbb{R}\right)$ and is therefore finite dimensional. In his thesis he further showed that for proper $X$ the vector spaces $\mathrm{H}^{1,0}\left(X^{\text {an }}\right)$ and $\mathrm{H}^{\operatorname{dim}(X), 0}\left(X^{\text {an }}\right)$ are finite dimensional. He did this by constructing injective maps $\mathrm{H}^{p, 0}\left(X^{\mathrm{an}}\right) \rightarrow \mathrm{H}^{0, p}\left(X^{\text {an }}\right)$ for $p=1$ and $p=\operatorname{dim}(X)$ [Jel16a, Proposition 3.4.11]. This was shown for all $p$ by Y. Liu when $K$ is a closed subfield of the completed algebraic closure of a field $K_{0}$, where $K_{0}$ is either a field of characteristic 0 or $K_{0}=k((t))$ for a finite field $k$ [Liu17a, Theorem 1.1]

Liu further showed that there are cycle class maps $\mathrm{CH}^{q}(X) \rightarrow \mathrm{H}^{q, q}\left(X^{\mathrm{an}}\right)[\operatorname{Liu} 17 \mathrm{~b}$, Theorem 1.1]. He additionally showed that for any smooth proper variety $X$ over $\mathbb{C}_{p}$ the space $\mathrm{H}^{1,1}\left(X^{\text {an }}\right)$ is finite dimensional [Liu17b, Theorem 1.9] and one dimensional when $X$ is a curve [Liu17b, Theorem 7.3 (2)]. His techniques include proving a weight filtration on the cohomology of the analytic de Rham complex.

Liu further constructed so called monodromy maps $N: \mathrm{H}^{p, q}\left(X^{\text {an }}\right) \rightarrow \mathrm{H}^{q, p}\left(X^{\text {an }}\right)$ for $p \geq q$ and conjectures that these are isomorphisms when $X$ is a smooth proper variety over an algebraically closed, non-archimedean field whose residue field is the algebraic closure of a finite field [Liu17a, Conjecture 5.2]. He showed that the map $N: \mathrm{H}^{1,0}\left(X^{\text {an }}\right) \rightarrow \mathrm{H}^{0,1}\left(X^{\text {an }}\right)$ is an isomorphism under the following assumptions: Firstly, he assumed that $X$ is the base change of a smooth proper variety $X_{0}$ over a field $K_{0}$ that is a finite extension of $\mathbb{Q}_{p}$ or $k((t))$ for a finite field $k$. Further he supposes that $X_{0}$ admits a proper strictly semistable model over $K_{0}^{\circ}$ [Liu17a, Theorem 1.1]. His techniques use arithmetic geometry, in particular the weight spectral sequence. Theorem A implies Liu's conjecture for curves in full generality (see Theorem 4.9).

For Mumford curves V. Wanner and the author showed that Poincaré duality holds over any complete non-archimedean algebraically closed field $K$ [JW18, Theorem 1]. The techniques in their work are tropical. They apply previous work by K. Shaw, J. Smacka 
and the author on Poincaré duality for smooth tropical varieties [JSS15]. Wanner and the author also obtained a Poincaré duality result for certain open subsets of $X^{\text {an }}$ for arbitrary smooth projective curves $X$ [JW18, Theorem 2]. In Section 4.2 we generalize this result to open subsets which form a basis of the topology of $X^{\text {an }}$ for any smooth curve $X$ that satisfies the conditions of Theorem A. The mentioned basis of the topology consists of so called strictly simple open subsets (see Definition 4.10). Our proof crucially relies on [JW18, Theorem 2].

Theorem C (Theorem 4.12). Let $X$ be a smooth projective curve over $K$ that satisfies Poincaré duality. Let $U$ be a strictly simple open subset of $X^{\text {an }}$ and denote by $k:=\# \partial U$ the finite number of boundary points. Then we have

$$
h^{p, q}(U)= \begin{cases}1 & \text { if }(p, q)=(0,0) \\ k-1 & \text { if }(p, q)=(1,0) \\ 0 & \text { else. }\end{cases}
$$

1.2. Notation and notions in other papers. Note that in [Jel16b] and [Jel16a] the author considers cohomology with respect to $d^{\prime}$ instead of $d^{\prime \prime}$. Therefore, when translating results to our setting, one has to flip the bigrading.

1.3. Techniques and outline of the paper. We study tropical Dolbeault cohomology by relating it to the cohomology of the sheaf $\mathcal{K}:=\operatorname{ker}\left(d^{\prime} d^{\prime \prime}: \mathcal{A}^{0,0} \rightarrow \mathcal{A}^{1,1}\right)$. The key new ingredient that establishes this relation is a short exact sequence, which might be thought of as a non-archimedean analogue of the exponential sequence in complex geometry. A tropical analogue of this short exact sequence was introduced by G. Mikhalkin and I. Zharkov to define 1-forms on tropical curves [MZ08]. We prove a non-archimedean version in Proposition 3.10. The terms of the associated long exact sequence include $\mathrm{H}^{1,0}\left(X^{\text {an }}\right), \mathrm{H}^{0,1}\left(X^{\text {an }}\right), \mathrm{H}^{1,1}\left(X^{\text {an }}\right)$ and $\mathrm{H}^{1}\left(X^{\text {an }}, \mathcal{K}\right)$. This sequence implies that Poincaré duality holds for $X$ if and only if $\mathrm{H}^{1}\left(X^{\text {an }}, \mathcal{K}\right)$ is one-dimensional and that $\mathrm{H}^{1,1}\left(X^{\text {an }}\right)$ is finite dimensional if and only if $\mathrm{H}^{1}\left(X^{\text {an }}, \mathcal{K}\right)$ is.

Our technique to calculate $\mathrm{H}^{1}\left(X^{\text {an }}, \mathcal{K}\right)$ involves Thuillier's sheaf of harmonic functions $\mathcal{H}_{X}$, which naturally contains $\mathcal{K}$ as a subsheaf. Using Thuillier's notion of lisse functions and 1-forms we show that $\mathrm{H}^{1}\left(X^{\mathrm{an}}, \mathcal{H}_{X}\right)$ is one dimensional. By [Thu05, Lemme 2.3.22] and [Wan16, Theorem 5.2.4] the quotient $\mathcal{H}_{X} / \mathcal{K}$ is a finite sum of skyscraper sheaves and vanishes if and only if the conditions of Theorem A are satisfied (see Definition 3.3 and Proposition 3.5).

This enables us to prove Theorem A using the above mentioned exact sequence and our calculation of the cohomology $\mathrm{H}^{1}\left(X^{\text {an }}, \mathcal{H}_{X}\right)$. For the proof of Theorem $\mathrm{B}$ we need to use that $\mathrm{H}^{0}\left(X^{\text {an }}, \mathcal{H}_{X} / \mathcal{K}\right)$ may have infinite dimension when the residue field of $K$ is $\mathbb{C}$.

To prove Theorem $\mathrm{C}$ we use Theorem A, a Mayer-Vietoris argument and a result by Wanner and the author [JW18, Theorem 2] to show that if $X$ satisfies the conditions of Theorem A, open subsets of $X^{\text {an }}$ satisfy a local version of Poincaré duality (see Theorem 4.7). While Mayer-Vietoris sequences are normally used to pass from local results to global results, we use our global result from Theorem A and the fact that we already have local results at all but finitely many points to deduce local results at all points. We then use results on the local structure of non-archimedean curves by M. Baker, S. Payne and J. Rabinoff [BPR13] and identification of tropical Dolbeault cohomology with singular cohomology to prove Theorem C.

Acknowledgements. The author would like to thank Walter Gubler, Klaus Künnemann and Veronika Wanner for reading previous drafts of this work and providing useful 
P. JELL

comments. Further he would like to thank Yifeng Liu, Johannes Rau and Kristin Shaw for helpful suggestions. Finally the author would like to thank the referee for their very precise report and helpful suggestions, which helped a lot in clarifying the presentation of the paper.

\section{PReliminaries}

Throughout this paper, $K$ will be an algebraically closed field that is complete with respect to a non-archimedean absolute value, which is assumed to be non-trivial. We denote its valuation ring by $K^{\circ}$ and its residue field by $\widetilde{K}$.

Further $X$ will be a curve over $K$, by which we mean an irreducible, reduced, separated, one-dimensional $K$-scheme of finite type.

2.1. Analytification. To the curve $X$, one can associate an analytic space $X^{\text {an }}$ over $K$ in the sense of Berkovich [Ber90]. We will merely concern ourselves with the underlying topological space of $X^{\text {an }}$, which is a paracompact Hausdorff space. This topological space is homotopy equivalent to a finite one-dimensional simplicial complex. If $X$ is projective, then $X^{\text {an }}$ is further compact.

The points in $X^{\text {an }}$ can be classified into four types [Thu05, p. 27] (extending results by Berkovich [Ber90, p. 19]). A point $x \in X^{\text {an }}$ is of type II if the residue field $\widetilde{\mathscr{H}}(x)$ of the completed residue field $\mathscr{H}(x)$ at $x$ is an extension of $\widetilde{K}$ of transcendence degree 1 . We denote by $C_{x}$ the smooth projective $\widetilde{K}$-curve with function field $\widetilde{\mathscr{H}}(x)$.

2.2. Thuillier's sheaf of harmonic functions. We recall Thuillier's notion of lisse functions, 1-forms and harmonic functions on $X^{\text {an }}$ for a smooth curve $X$ and their properties.

Definition 2.1 ([Thu05]). Let $U \subset X^{\text {an }}$ be an open subset. We denote the space of lisse functions in the sense of [Thu05, Section 3.2.1] by $\mathrm{L}^{0}(U)$.

The space $\mathrm{L}^{1}(U)$ is defined as the space of measures on $U$ with discrete support that is contained in the set of type II and III points of $X^{\text {an }}$.

We denote by $\mathcal{H}_{X}(U)$ the kernel of the operator $\mathrm{dd}^{\mathrm{c}}: \mathrm{L}^{0}(U) \rightarrow \mathrm{L}^{1}(U)$ constructed in [Thu05, Théorème 3.2.10]. The elements in $\mathcal{H}_{X}(U)$ are called harmonic functions on $U$.

Remark 2.2. Roughly speaking, a function is lisse if it can be locally written as the pullback along the retraction of a piecewise linear function on some skeleton. It is shown in [Thu05] that both $U \mapsto \mathrm{L}^{0}(U)$ and $U \mapsto \mathrm{L}^{1}(U)$ define sheaves in the Berkovich topology of $X^{\text {an }}$ and that $\mathrm{dd}^{\mathrm{c}}$ is a morphism of sheaves, which implies that $U \mapsto \mathcal{H}_{X}(U)$ also defines a sheaf on $X^{\text {an }}$. We consider the sheaves $L^{0}$ and $L^{1}$ as sheaves in the Berkovich topology. Thuillier denotes these by $\mathcal{A}^{0}$ and $\mathcal{A}^{1}$, as opposed to the corresponding sheaves in the $G$-topology, which he denotes by $A^{0}$ and $A^{1}$. To avoid confusion with sheaves of forms $\mathcal{A}^{p, q}$, we differ from Thuillier's notation here.

Also note that this is not the definition of $\mathcal{H}_{X}$ that Thuillier gives, but is equivalent by [Thu05, Corollaire 3.2.11].

Recall that a sheaf $\mathcal{F}$ on a topological space $X$ is soft if for every closed subset $B$ of $X$ the map $\mathcal{F}(X) \rightarrow \underset{U \supset B}{\lim _{U \supset B}} \mathcal{F}(U)$, where $U$ are open subsets of $X$ containing $B$, is surjective.

Lemma 2.3. The sheaf $\mathrm{L}^{0}$ is soft.

Proof. Let $B \subset X^{\text {an }}$ be a closed subset. Take $f \in \mathrm{L}^{0}(U)$ for an open subset $U$ containing $B$. Take $U_{1}$ to be another neighborhood of $B$ that furthermore satisfies $\bar{U}_{1} \subset U$. Let 
$U_{2}:=U \backslash B$ and $\mathcal{U}:=\left\{U_{1}, U_{2}\right\}$. Using the identical arguments as in the proof of [Thu05, Lemme 3.3.5] we find a locally finite refinement $\mathcal{V}$ of $\mathcal{U}$ and for all $V \in \mathcal{V}$ a lisse function $f_{V} \in L^{0}(U)$ with compact support, such that $\operatorname{supp}\left(f_{V}\right) \subset \bar{V}$ and $\sum_{V \in \mathcal{V}} f_{V}=f$. Now taking $g=\sum_{V \in \mathcal{V}, V \subset U_{1}} f_{V}$ gives a lisse function on $U_{1}$ that has support away from the boundary of $U_{1}$. Thus $g$ may be extended by zero to a lisse function on all of $X$. Further it agrees with $f$ on a neighborhood of $B$, as was required.

2.3. Tropical Dolbeault cohomology. Chambert-Loir and Ducros introduced a double complex $\left(\mathcal{A}^{\bullet, \bullet}, d^{\prime}, d^{\prime \prime}\right)$ of sheaves of smooth real differential forms on Berkovich analytic spaces [CLD12]. If $X$ is a curve over a non-archimedean field, then we have $\mathcal{A}^{p, q}=0$ unless $0 \leq p \leq 1$ and $0 \leq q \leq 1$. Elements of $\mathcal{A}^{0,0}$ are functions. We call these functions smooth and write $C^{\infty}:=\mathcal{A}^{0,0}$.

Definition 2.4. For all $p, q$ we denote $\mathcal{A}^{p, q, \mathrm{cl}}:=\operatorname{ker}\left(d^{\prime \prime}: \mathcal{A}^{p, q} \rightarrow \mathcal{A}^{p, q+1}\right)$.

Furthermore we denote $\mathcal{K}:=\operatorname{ker}\left(d^{\prime} d^{\prime \prime}: C^{\infty} \rightarrow \mathcal{A}^{1,1}\right)$ and call this the sheaf of affine functions.

We also define the tropical Dolbeault cohomology of $X$ to be

$$
\mathrm{H}^{p, q}\left(X^{\mathrm{an}}\right):=\frac{\mathcal{A}^{p, q, \mathrm{cl}}\left(X^{\mathrm{an}}\right)}{d^{\prime \prime}\left(\mathcal{A}^{p, q-1}\left(X^{\mathrm{an}}\right)\right)} .
$$

Remark 2.5. We have $\mathrm{H}^{p, q}\left(X^{\text {an }}\right) \cong \mathrm{H}^{q}\left(X^{\text {an }}, \mathcal{A}^{p, 0, \text { cl }}\right)$ by [Jel16a, Corollary 3.4.6] and $\mathcal{A}^{0,0, \mathrm{cl}} \cong \mathbb{R}$ by [Jel16a, Lemma 3.4.5]. This implies $\mathrm{H}^{0, q}\left(X^{\mathrm{an}}\right) \cong \mathrm{H}_{\text {sing }}^{q}\left(X^{\text {an }}, \mathbb{R}\right)$, the $q$-th singular cohomology of $X^{\text {an }}$ with values in $\mathbb{R}$.

The double complex $\mathcal{A}^{\bullet \bullet \bullet}$ admits a wedge-product and if $X$ is of dimension one, canonical integration of $(1,1)$-forms with compact support. A version of Stokes' theorem ensures that the induced pairing descends to cohomology [Gub16, Theorem 5.17]. In particular, we obtain for a projective curve $X$ pairings

$$
\begin{aligned}
& \mathrm{H}^{0,0}\left(X^{\mathrm{an}}\right) \times \mathrm{H}^{1,1}\left(X^{\mathrm{an}}\right) \rightarrow \mathbb{R} \text { and } \\
& \mathrm{H}^{1,0}\left(X^{\mathrm{an}}\right) \times \mathrm{H}^{0,1}\left(X^{\mathrm{an}}\right) \rightarrow \mathbb{R} .
\end{aligned}
$$

Definition 2.6. We say that Poincaré duality holds for $X$ if the pairings in (2.1) are perfect pairings.

\section{Harmonic functions, affine functions and $(1,1)$-tropical Dolbeault COHOMOLOGY}

In this section we prove the existence of certain exact sequences relating the cohomology of the sheaves $\mathcal{K}$ and $\mathcal{H}_{X}$ to tropical Dolbeault cohomology.

3.1. Sheaf cohomology of $\mathcal{H}_{X}$. In this section $X$ is a smooth curve over $K$.

Lemma 3.1. Let $X$ be a smooth curve. The sequence

$$
0 \rightarrow \mathcal{H}_{X} \rightarrow \mathrm{L}^{0} \stackrel{\mathrm{dd}^{\mathrm{c}}}{\rightarrow} \mathrm{L}^{1} \rightarrow 0
$$

is exact.

Proof. Exactness at $\mathcal{H}_{X}$ and $\mathrm{L}^{0}$ follows from the definition. We check exactness at $\mathrm{L}^{1}$ on stalks. Take $x \in X^{\text {an }}$. If $x$ is of type I or IV, then $\mathrm{L}_{x}^{1}$ is zero, so we are done.

If $x$ is of type II or III, the real vector space $\mathrm{L}_{x}^{1}$ is spanned by $\delta_{x}$, the Dirac measure at $x$. Now take any other type II or III point $y \in X^{\text {an }}$. Denote by $\bar{X}$ the canonical smooth compactification of $X$. By [Thu05, Proposition 3.3.7] there exists a function $g \in \mathrm{L}^{0}\left(\bar{X}^{\mathrm{an}}\right)$ such that $\mathrm{dd}^{\mathrm{c}} g=\delta_{x}-\delta_{y}$. Now the stalk $g_{x}$ of $\left.g\right|_{X^{\text {an }}}$ at $x$ satisfies $\mathrm{dd}^{\mathrm{c}}\left(g_{x}\right)=\delta_{x}$. 
Proposition 3.2. Let $X$ be a smooth projective curve. Then there are canonical isomorphisms $\mathrm{H}^{0}\left(X^{\text {an }}, \mathcal{H}_{X}\right) \cong \mathbb{R}$ and $\mathrm{H}^{1}\left(X^{\text {an }}, \mathcal{H}_{X}\right) \cong \mathbb{R}$.

Proof. Since $X^{\text {an }}$ is a paracompact and Hausdorff topological space, soft sheaves on $X^{\text {an }}$ are acyclic. Thus we have $\mathrm{H}^{1}\left(X^{\mathrm{an}}, \mathrm{L}^{0}\right)=0$ by Lemma 2.3. Looking at the long exact sequence in cohomology associated to (3.1) we now find that

$$
0 \rightarrow \mathrm{H}^{0}\left(X^{\text {an }}, \mathcal{H}_{X}\right) \rightarrow \mathrm{L}^{0}\left(X^{\text {an }}\right) \rightarrow \mathrm{L}^{1}\left(X^{\text {an }}\right) \rightarrow \mathrm{H}^{1}\left(X^{\text {an }}, \mathcal{H}_{X}\right) \rightarrow 0
$$

is exact. Thus, we have to calculate the kernel and cokernel of $d^{c}: \mathrm{L}^{0}\left(X^{\text {an }}\right) \rightarrow \mathrm{L}^{1}\left(X^{\text {an }}\right)$. The kernel is $\mathcal{H}_{X}\left(X^{\text {an }}\right)$, which consists of constant functions by the maximum principle [Thu05, Proposition 3.1.1].

The isomorphism of the cokernel with $\mathbb{R}$ is given by total mass

$$
\int: \mathrm{L}^{1}\left(X^{\mathrm{an}}\right) \rightarrow \mathbb{R} ; \mu \mapsto \int 1 \mu .
$$

For $f \in \mathrm{L}^{0}\left(X^{\mathrm{an}}\right)$ we have $\int 1 \mathrm{dd}^{\mathrm{c}} f=\int f \mathrm{dd}^{\mathrm{c}} 1=0$ by [Thu05, Proposition 3.2.12]. Now let $\mu=\sum_{i=0}^{k} \lambda_{i} \delta_{x_{i}} \in \mathrm{L}^{1}\left(X^{\text {an }}\right)$ be a discrete measure of mass 0 . Then by [Thu05, Proposition 3.3.7] there exist functions $g_{i}:=g_{x_{i} x_{0}} \in \mathrm{L}^{0}\left(X^{\text {an }}\right)$ such that $\operatorname{dd}^{\mathrm{c}}\left(g_{i}\right)=\delta_{x_{i}}-$ $\delta_{x_{0}}$. Write $g:=\sum_{i=1}^{k} \lambda_{i} g_{i}$. We obtain $\mathrm{dd}^{\mathrm{c}}(g)=\sum_{i=1}^{k} \lambda_{i} \delta_{x_{i}}+\left(\sum_{i=1}^{k}-\lambda_{i}\right) \delta_{x_{0}}$. Since $\mu$ was of mass zero, we have $\lambda_{0}=\sum_{i=1}^{k}-\lambda_{i}$ which shows $\mu=\mathrm{dd}^{\mathrm{c}} g$. Thus integration induces an isomorphism $\mathrm{L}^{1}\left(X^{\mathrm{an}}\right) / \mathrm{dd}^{\mathrm{c}} \mathrm{L}^{0}\left(X^{\text {an }}\right)$ with $\mathbb{R}$, which is precisely what we wanted to show.

3.2. Relation between $\mathcal{H}_{X}$ and $\mathcal{K}$. In this section $X$ is again a smooth curve over $K$.

Definition 3.3. We write $G(X)$ for the set of type II points $x$ of $X^{\text {an }}$ where the genus of the $\widetilde{K}$-curve $C_{x}$ is positive. We define the sheaf

$$
\mathcal{S}_{X}:=\bigoplus_{x \in G(X)} \iota_{x, *}\left(\operatorname{Pic}^{0}\left(C_{x}\right) \otimes_{\mathbb{Z}} \mathbb{R}\right),
$$

where $\iota_{x}:\{x\} \rightarrow X^{\text {an }}$ denotes the inclusion, and the real vector space

$$
S_{X}:=\bigoplus_{x \in G(X)}\left(\operatorname{Pic}^{0}\left(C_{x}\right) \otimes_{\mathbb{Z}} \mathbb{R}\right)=\mathcal{S}_{X}\left(X^{\mathrm{an}}\right) .
$$

Remark 3.4. The set $G(X)$ is finite [BPR13, Remark 4.18]. We could let the sums in (3.2) and (3.3) run over all type II points on $X^{\text {an }}$, since if $x \notin G(X)$ we have $C_{x} \cong \mathbb{P}_{\widetilde{K}}^{1}$ and consequently $\operatorname{Pic}^{0}\left(C_{x}\right)=0$.

Definition 3.5. A model $\mathcal{X}$ of $X$ over $K^{\circ}$ is called semistable if the special fiber $\mathcal{X}_{s}:=$ $\mathcal{X} \times K^{\circ} \widetilde{K}$ has only ordinary double points as singularities. It is called strictly semistable if all irreducible components of $\mathcal{X}_{s}$ are smooth.

Remark 3.6. Let $X$ be a smooth projective curve over $K$ and $\mathcal{X}$ a semistable model of $X$ over $K^{\circ}$. There exists a canonical reduction map red: $X^{\text {an }} \rightarrow \mathcal{X}_{s}$.

Let $C$ be an irreducible component of $\mathcal{X}_{s}$ with generic point $\eta$. Then $\eta$ has a unique preimage under red [BPR13, Theorem 4.6]. This preimage, denoted by $x$, is a type II point such that $C_{x} \cong C^{\prime}$, where $C^{\prime}$ is the normalization of $C$. For all points of type II that do not appear in this way we have $C_{x} \cong \mathbb{P}_{\widetilde{K}}^{1}$ [BPR13, Remarks $4.17 \& 4.18$ ].

In other words

$$
S_{X}=\bigoplus_{\text {irrd. comp. } C \text { of } \mathcal{X}_{s}} \operatorname{Pic}^{0}\left(C^{\prime}\right) \otimes_{\mathbb{Z}} \mathbb{R}
$$


for every semistable model $\mathcal{X}$ of $X$.

Remark 3.7. Thuillier defines a map $\mathcal{H}_{X} \rightarrow \mathcal{S}_{X}$ as follows [Thu05, Proof of Lemma 2.3.22]: The tangent directions at a type II point $x \in X^{\text {an }}$ are in canonical bijection with $C_{x}(\widetilde{K})$. Thus for a harmonic function $h$ defined in a neighborhood of $x$ there is an associated $\mathbb{R}$-divisor $\operatorname{div}_{x}(h):=\sum d_{x, v_{p}}(h) \cdot p \in \operatorname{Div}\left(C_{x}\right) \otimes \mathbb{R}$, where $v_{p}$ denotes the tangent direction associated to $p$ and $d_{x, v_{p}}(h)$ denotes the slope of $h$ in the direction of $v_{p}$. Since $h$ is harmonic at $x$, we have $\operatorname{div}_{x}(h) \in \operatorname{Div}^{0}\left(C_{x}\right) \otimes \mathbb{R}$. We thus obtain a map $\mathcal{H}_{X, x} \rightarrow \operatorname{Pic}^{0}\left(C_{x}\right) \otimes \mathbb{R}=\mathcal{S}_{X, x}$ on stalks by mapping $h$ to the class of $\operatorname{div}_{x}(h)$ in $\operatorname{Pic}^{0}\left(C_{x}\right) \otimes \mathbb{R}$. These maps glue to define a map $\mathcal{H}_{X} \rightarrow \mathcal{S}_{X}$.

Proposition 3.8. Let $X$ be a smooth curve. The sequence of sheaves

$$
0 \rightarrow \mathcal{K} \rightarrow \mathcal{H}_{X} \rightarrow \mathcal{S}_{X} \rightarrow 0
$$

is exact, where the map $\mathcal{H}_{X} \rightarrow \mathcal{S}_{X}$ is the one constructed in Remark 3.7.

Proof. It is shown in [Thu05, Lemme 2.3.22] that

$$
0 \rightarrow \mathbb{R} \log \left|\mathcal{O}_{X}^{\times}\right| \rightarrow \mathcal{H}_{X} \rightarrow \mathcal{S}_{X} \rightarrow 0
$$

is exact. Here $\mathbb{R} \log \left|\mathcal{O}_{X}^{\times}\right|$denotes the sheaf of real-valued functions that can be locally written as $\sum_{i=1}^{k} \lambda_{i} \cdot \log \left|f_{i}\right|$ for $\lambda_{i} \in \mathbb{R}$ and $f_{i}$ invertible functions. It is then shown in [Wan16, Theorem 5.2.4] that $\mathcal{K} \cong \mathbb{R} \log \left|\mathcal{O}_{X}^{\times}\right|$.

Corollary 3.9. Let $X$ be a smooth projective curve. The sequence

$$
0 \rightarrow S_{X} \rightarrow \mathrm{H}^{1}\left(X^{\mathrm{an}}, \mathcal{K}\right) \rightarrow \mathrm{H}^{1}\left(X^{\mathrm{an}}, \mathcal{H}_{X}\right) \rightarrow 0
$$

of real vector spaces is exact. In particular, we have $\operatorname{dim} \mathrm{H}^{1}\left(X^{\mathrm{an}}, \mathcal{K}\right)=\operatorname{dim} S_{X}+1$.

Proof. We consider the long exact cohomology sequence associated to (3.5):

$$
\begin{aligned}
0 \rightarrow \mathrm{H}^{0}\left(X^{\mathrm{an}}, \mathcal{K}\right) & \rightarrow \mathrm{H}^{0}\left(X^{\mathrm{an}}, \mathcal{H}_{X}\right) \rightarrow \mathrm{H}^{0}\left(X^{\mathrm{an}}, \mathcal{S}_{X}\right) \rightarrow \\
\mathrm{H}^{1}\left(X^{\mathrm{an}}, \mathcal{K}\right) & \rightarrow \mathrm{H}^{1}\left(X^{\mathrm{an}}, \mathcal{H}_{X}\right) \rightarrow \mathrm{H}^{1}\left(X^{\mathrm{an}}, \mathcal{S}_{X}\right) \rightarrow \ldots
\end{aligned}
$$

Now we have $H^{0}\left(X^{\text {an }}, \mathcal{K}\right) \cong \mathbb{R}$ by [Jel16a, Theorem 3.2.61] and $H^{0}\left(X^{\text {an }}, \mathcal{H}_{X}\right) \cong \mathbb{R}$ by Proposition 3.2. This implies that the map $\mathrm{H}^{0}\left(X^{\text {an }}, \mathcal{H}_{X}\right) \rightarrow \mathrm{H}^{0}\left(X^{\text {an }}, \mathcal{S}_{X}\right)$ is zero. Further we have $\mathrm{H}^{0}\left(X^{\text {an }}, \mathcal{S}_{X}\right)=S_{X}$, and $\mathrm{H}^{1}\left(X^{\text {an }}, \mathcal{S}_{X}\right)=0$ since $\mathcal{S}$ is a finite sum of skyscraper sheaves. This yields the claimed short exact sequence.

The last statement follows since $\mathrm{H}^{1}\left(X^{\text {an }}, \mathcal{H}_{X}\right) \cong \mathbb{R}$ by Proposition 3.2.

3.3. Relation between $\mathrm{H}^{1,1}\left(X^{\text {an }}\right)$ and $\mathrm{H}^{1}\left(X^{\text {an }}, \mathcal{K}\right)$. Recall that we defined $\mathcal{A}^{1,0, \mathrm{cl}}:=$ $\operatorname{ker}\left(d^{\prime \prime}: \mathcal{A}^{1,0} \rightarrow \mathcal{A}^{1,1}\right)$.

Proposition 3.10. The sequence

$$
0 \rightarrow \underline{\mathbb{R}} \rightarrow \mathcal{K} \stackrel{d^{\prime}}{\rightarrow} \mathcal{A}^{1,0, \mathrm{cl}} \rightarrow 0
$$

of sheaves on $X^{\text {an }}$ is exact.

Let us mention here that the tropical analogue of this sequence was introduced by Mikhalkin and Zharkov [MZ08, Definition 4.1]. Note here that the functions in $\mathcal{K}$ are precisely the ones that locally factor as an affine function through a tropicalization. 
P. JELL

Proof. Note that $d^{\prime}$ indeed maps $\mathcal{K}$ to $\mathcal{A}^{1,0, \text { cl }}$, since if $f \in \mathcal{K}$ we have $d^{\prime \prime} d^{\prime} f=-d^{\prime} d^{\prime \prime} f=0$. We have $\operatorname{ker}\left(d^{\prime}: C^{\infty} \rightarrow \mathcal{A}^{1,0}\right)=\underline{\mathbb{R}}$ by [Jel16b, Corollary 4.6]. Since $\underline{\mathbb{R}}$ is a subsheaf of $\mathcal{K}$ we obtain exactness at $\mathcal{K}$.

We check exactness at $\mathcal{A}^{1,0, \mathrm{cl}}$ on stalks. Let $x \in X^{\text {an }}$ and $\alpha \in \mathcal{A}^{1,0, \mathrm{cl}}(U)$ for an open subset $U$ of $X^{\text {an }}$ containing $x$. Then $d^{\prime} \alpha \in \mathcal{A}^{2,0}(U)=0$, thus by [Jel16b, Theorem 4.5] there exists an open subset $V \subset U$ containing $x$ and $f \in C^{\infty}(V)$ such that $d^{\prime} f=\alpha$. Since $d^{\prime} d^{\prime \prime} f=-d^{\prime \prime} \alpha=0$ we have $f \in \mathcal{K}(V)$. This shows exactness on the stalk of $x$ at $\mathcal{A}^{1,0, \mathrm{cl}}$.

Corollary 3.11. For a smooth projective curve $X$ we obtain the exact sequence

$$
0 \rightarrow \mathrm{H}^{1,0}\left(X^{\mathrm{an}}\right) \rightarrow \mathrm{H}^{0,1}\left(X^{\mathrm{an}}\right) \rightarrow \mathrm{H}^{1}\left(X^{\text {an }}, \mathcal{K}\right) \rightarrow \mathrm{H}^{1,1}\left(X^{\text {an }}\right) \rightarrow 0
$$

of real vector spaces.

Proof. The long exact sequence in cohomology associated to (3.6) reads

$$
\begin{aligned}
0 & \rightarrow \mathrm{H}^{0}\left(X^{\mathrm{an}}, \underline{\mathbb{R}}\right) \rightarrow \mathrm{H}^{0}\left(X^{\mathrm{an}}, \mathcal{K}\right) \rightarrow \mathrm{H}^{0}\left(X^{\mathrm{an}}, \mathcal{A}^{1,0, \mathrm{cl}}\right) \\
& \rightarrow \mathrm{H}^{1}\left(X^{\mathrm{an}}, \underline{\mathbb{R}}\right) \rightarrow \mathrm{H}^{1}\left(X^{\mathrm{an}}, \mathcal{K}\right) \rightarrow \mathrm{H}^{1}\left(X^{\mathrm{an}}, \mathcal{A}^{1,0, \mathrm{cl}}\right) \rightarrow \mathrm{H}^{2}\left(X^{\mathrm{an}}, \underline{\mathbb{R}}\right) \rightarrow \ldots
\end{aligned}
$$

Now using $\mathrm{H}^{0}\left(X^{\mathrm{an}}, \mathcal{K}\right) \cong \mathbb{R}\left[\right.$ Jel16a, Theorem 3.2.61], $\mathrm{H}^{q}\left(X^{\mathrm{an}}, \mathcal{A}^{p, 0, \mathrm{cl}}\right) \cong \mathrm{H}^{p, q}\left(X^{\mathrm{an}}\right)$ [Jel16a, Corollary 3.4.6], $\mathcal{A}^{0,0, \mathrm{cl}} \cong \mathbb{R}\left[\mathrm{Jel16b}\right.$, Lemma 3.4.5], and the fact that $X^{\text {an }}$ is homotopy equivalent to a finite one-dimensional simplicial complex, which implies $\mathrm{H}^{2}\left(X^{\text {an }}, \underline{\mathbb{R}}\right)=0$, we obtain

$$
0 \rightarrow \mathbb{R} \rightarrow \mathbb{R} \rightarrow \mathrm{H}^{1,0}\left(X^{\mathrm{an}}\right) \rightarrow \mathrm{H}^{0,1}\left(X^{\mathrm{an}}\right) \rightarrow \mathrm{H}^{1}\left(X^{\mathrm{an}}, \mathcal{K}\right) \rightarrow \mathrm{H}^{1,1}\left(X^{\text {an }}\right) \rightarrow 0 .
$$

Now by exactness the map $\mathbb{R} \rightarrow \mathrm{H}^{1,0}\left(X^{\text {an }}\right)$ is zero and we obtain the result.

\section{Poincaré Duality and finite dimensionality}

4.1. Poincaré duality and finite dimensionality for smooth projective curves. In this section, $X$ is a smooth projective curve over $K$.

We write $h^{p, q}\left(X^{\text {an }}\right):=\operatorname{dim}_{\mathbb{R}} \mathrm{H}^{p, q}\left(X^{\text {an }}\right)$ and call these numbers tropical Hodge numbers.

Lemma 4.1. The Poincaré duality pairings

$$
\begin{aligned}
& \mathrm{H}^{0,0}\left(X^{\mathrm{an}}\right) \times \mathrm{H}^{1,1}\left(X^{\mathrm{an}}\right) \rightarrow \mathbb{R} \text { and } \\
& \mathrm{H}^{1,0}\left(X^{\mathrm{an}}\right) \times \mathrm{H}^{0,1}\left(X^{\mathrm{an}}\right) \rightarrow \mathbb{R}
\end{aligned}
$$

are non-degenerate on the left, i.e. for all $\alpha \in \mathrm{H}^{p, 0}\left(X^{\mathrm{an}}\right) \backslash\{0\}$ there exists $\beta \in \mathrm{H}^{1-p, 1}\left(X^{\mathrm{an}}\right)$ such that $\langle\alpha, \beta\rangle \neq 0$.

In particular, Poincaré duality holds for $X$ if and only if $h^{1,1}\left(X^{\text {an }}\right)=1$ and $h^{1,0}\left(X^{\text {an }}\right)=$ $h^{0,1}\left(X^{\text {an }}\right)$.

Proof. We have that $\mathrm{H}^{0,0}\left(X^{\text {an }}\right)$ is the space of constant functions. Since the integration map $\mathcal{A}^{1,1}\left(X^{\text {an }}\right) \rightarrow \mathbb{R}$ is non-trivial, $\mathbb{R}$-linear and descends to cohomology, the first pairing is non-degenerate on the left.

By [Jel16a, Proposition 3.4.11] we have an injective map $J: \mathrm{H}^{1,0}\left(X^{\text {an }}\right) \rightarrow \mathrm{H}^{0,1}\left(X^{\text {an }}\right)$ with the property that $\int \alpha \wedge J \alpha \neq 0$ if $\alpha \neq 0$. This shows that the second pairing is non-degenerate on the left.

The last statement is a direct consequence of the fact that $h^{1,0}\left(X^{\text {an }}\right)$ is finite and $h^{0,0}\left(X^{\mathrm{an}}\right)=1$.

Theorem 4.2. For a smooth projective curve $X$, Poincaré duality holds if and only if $S_{X}=0$. 
Proof. If Poincaré duality holds, the injective map $\mathrm{H}^{1,0}\left(X^{\text {an }}\right) \rightarrow \mathrm{H}^{0,1}\left(X^{\text {an }}\right)$ from Corollary 3.11 is an isomorphism. Thus $\mathrm{H}^{1}\left(X^{\text {an }}, \mathcal{K}\right) \rightarrow \mathrm{H}^{1,1}\left(X^{\text {an }}\right)$ is also an isomorphism. Since the latter space has dimension 1 again by Poincaré duality, so does $\mathrm{H}^{1}\left(X^{\text {an }}, \mathcal{K}\right)$. By Corollary 3.9 we have $\operatorname{dim} S_{X}=\operatorname{dim} H^{1}\left(X^{\text {an }}, \mathcal{K}\right)-1$ and hence $S_{X}=0$.

Conversely if $S_{X}=0$, then again by Corollary 3.9 we have $\mathrm{H}^{1}\left(X^{\text {an }}, \mathcal{K}\right) \cong \mathbb{R}$. Since $h^{1,1}\left(X^{\text {an }}\right) \geq 1$, the surjective map $\mathrm{H}^{1}\left(X^{\text {an }}, \mathcal{K}\right) \rightarrow \mathrm{H}^{1,1}\left(X^{\text {an }}\right)$ from Corollary 3.11 is an isomorphism. Thus the injective map $\mathrm{H}^{1,0}\left(X^{\text {an }}\right) \rightarrow \mathrm{H}^{0,1}\left(X^{\text {an }}\right)$ from Corollary 3.11 is also an isomorphism, which shows that we have Poincaré duality by Lemma 4.1.

Corollary 4.3. Let $K$ be an algebraically closed non-archimedean field whose residue field is the algebraic closure of a finite field. Then Poincaré duality holds for smooth projective curves $X$ over $K$.

Proof. We have to show that for any point $x \in X^{\text {an }}$ of type II with associated $\widetilde{K}$-curve $C_{x}$ the group $\operatorname{Pic}^{0}\left(C_{x}\right)$ is torsion. Now $\operatorname{Pic}^{0}\left(C_{x}\right)$ is the set of $\widetilde{K}$-points of an abelian variety $J_{x}$, namely the Jacobian of $C_{x}$. Any element of $J_{x}(\widetilde{K})$ is defined over some finite subfield of $\widetilde{K}$, thus is torsion.

Recall the subset $G(X)$ of $X^{\text {an }}$, defined in 3.3, which is the finite set of type II points of positive genus.

Theorem 4.4. Let $X$ be a smooth projective curve over $K$ and let the residue field of $K$ be $\mathbb{C}$. Then $\mathrm{H}^{1,1}(X)$ is finite dimensional if and only if $G(X)=\emptyset$.

Proof. If $G(X)=\emptyset$, then $S_{X}=0$ and thus $X$ satisfies Poincaré duality by Theorem A (or equivalently by [JW18, Theorem 2]). Since $\mathrm{H}^{0,0}\left(X^{\text {an }}\right) \cong \mathbb{R}$, we then have $\mathrm{H}^{1,1}\left(X^{\text {an }}\right) \cong \mathbb{R}$, thus $\mathrm{H}^{1,1}\left(X^{\text {an }}\right)$ is in particular finite dimensional.

We now show that $G(X) \neq \emptyset$ implies that $\mathrm{H}^{1,1}\left(X^{\text {an }}\right)$ is not finite dimensional. The vector space $\mathrm{H}^{0,1}\left(X^{\text {an }}\right)$ is finite dimensional by [Jel16b, Theorem 4.9]. By Corollary 3.11 we thus have to show that $\mathrm{H}^{1}\left(X^{\text {an }}, \mathcal{K}\right)$ is not finite dimensional if $G(X) \neq \emptyset$. By Corollary 3.9 it is thus enough to show that $S_{X}$ is not finite dimensional when $G(X) \neq \emptyset$. If $x \in G(X)$, then $C_{x}$ is a smooth projective curve of positive genus $g$ over $\mathbb{C}$. Thus $\operatorname{Pic}^{0}\left(C_{x}\right) \cong \mathbb{C}^{g} / \Gamma$ for some lattice $\Gamma$. In particular $\operatorname{Pic}^{0}\left(C_{x}\right)$ is not of finite rank as an abelian group, thus $\operatorname{dim}_{\mathbb{R}}\left(\operatorname{Pic}^{0}\left(C_{x}\right) \otimes_{\mathbb{Z}} \mathbb{R}\right.$ ) is not finite (since the tensor product is taken over $\mathbb{Z})$. Therefore $\operatorname{dim}_{\mathbb{R}} S_{X}$ is not finite.

Remark 4.5. We want to remark that curves with $G(X) \neq \emptyset$ actually exist. One way to see this is that any curve over $K$ with $G(X)=\emptyset$ is either $\mathbb{P}_{K}^{1}$ or a Mumford curve [JW18, Proposition 2.26 \& Theorem 2.28]. More concretely, by Remark 3.6, any curve of positive genus with good reduction has non-empty $G(X)$.

4.2. From global Poincaré duality to local Poincaré duality. In this section $X$ is a smooth curve over a non-archimedean field $K$.

We now change our perspective on Poincaré duality by considering forms with compact support. For any open subset $U \subset X^{\text {an }}$ we define $\mathcal{A}_{c}^{p, q}(U)$ to be the vector subspace of $\mathcal{A}^{p, q}(U)$ of forms with compact support. We define

$$
\mathrm{H}_{c}^{p, q}(U):=\frac{\operatorname{ker}\left(d^{\prime \prime}: \mathcal{A}_{c}^{p, q}(U) \rightarrow \mathcal{A}_{c}^{p, q+1}(U)\right)}{\operatorname{im}\left(d^{\prime \prime}: \mathcal{A}_{c}^{p, q-1}(U) \rightarrow \mathcal{A}_{c}^{p, q}(U)\right)} .
$$

Integration and the wedge product induce a pairing

$$
\mathcal{A}^{p, q}(U) \times \mathcal{A}_{c}^{1-p, 1-q}(U) \rightarrow \mathbb{R} .
$$


This descends to a pairing on cohomology by a suitable version of Stokes' theorem [Gub16, Theorem 5.17]. We will from now on consider the induced map

$$
\mathrm{PD}_{U}: \mathrm{H}^{p, q}(U) \rightarrow \mathrm{H}_{c}^{1-p, 1-q}(U)^{*}:=\operatorname{Hom}_{\mathbb{R}}\left(\mathrm{H}_{c}^{1-p, 1-q}(U), \mathbb{R}\right) .
$$

We say that $U$ satisfies Poincaré duality $(\mathrm{PD})$ if $\mathrm{PD}_{U}$ is an isomorphism for all $p, q$. If $X^{\text {an }}$ is compact, this agrees with Definition 2.6 since $\mathrm{H}^{p, q}\left(X^{\text {an }}\right)=\mathrm{H}_{c}^{p, q}\left(X^{\text {an }}\right)$ in that case. Note that $U$ satisfies PD if it is disjoint from $G(X)$ by [JW18, Theorem 2]. We will crucially use this fact.

Lemma 4.6. Let $U$ be an open subset of $X^{\text {an }}$.

i) Let $U=U_{1} \cup U_{2}$ and write $U_{12}=U_{1} \cap U_{2}$. Then if three of the four sets $U, U_{1}, U_{2}, U_{12}$ satisfy $\mathrm{PD}$, so does the fourth.

ii) Let $U=\bigcup_{i=1}^{k} U_{k}$ be a disjoint union of open subsets and suppose that $U$ satisfies PD. Then for every subset $J \subset\{1, \ldots, k\}$ the subset $\bigcup_{j \in J} U_{j}$ satisfies PD.

Proof. To prove $i$ ) the standard arguments from differential geometry work in our setting. See for instance the book by Bott and Tu on differential forms [BT82, Lemma 5.6]. Since the sheaves $\mathcal{A}^{p, q}$ admit partitions of unity [CLD12, Proposition 3.3.6] both $\mathrm{H}^{p, q}$ and $\mathrm{H}_{c}^{p, q}$ satisfy Mayer-Vietoris-sequences [Jel16a, Appendix A.1]. Dualizing the Mayer-Vietorissequence for $\mathrm{H}_{c}^{p, q}$, we see that $\mathrm{PD}$ induces a morphism of complexes between the MayerVietoris-sequence for $\mathrm{H}^{p, q}$ and the one for $\mathrm{H}_{c}^{1-p, 1-q^{*}}$. The claim now follows from the five lemma.

For $i i$ ), notice that we have $\mathrm{PD}_{U}=\bigoplus_{i=1}^{k} \mathrm{PD}_{U_{i}}$. Thus if $\mathrm{PD}_{U}$ is an isomorphism, so is $\mathrm{PD}_{\bigcup_{j \in J} U_{j}}=\bigoplus_{j \in J} \mathrm{PD}_{U_{j}}$.

Theorem 4.7. Let $X$ be a smooth curve and $U \subset X^{\text {an }}$ an open subset. Assume further that $S_{X}=0$. Then $U$ satisfies $\mathrm{PD}$.

Proof. Replacing $X$ by its canonical smooth compactification, we may assume that $X$ is projective. Then we still have $S_{X}=0$.

We first treat the case where $U$ contains $G(X)$. Recall that $G(X)$ is the finite set of type II points such that $C_{x}$ has positive genus. Now $X^{\text {an }}$ satisfies PD by Theorem 4.2 and $X^{\text {an }} \backslash G(X)$ and $U \backslash G(X)$ do so by [JW18, Theorem 2], since they do not contain any type II points of positive genus. Then using Lemma $4.6 i$ ) with $U=X^{\text {an }}, U_{1}=U$ and $U_{2}=X^{\text {an }} \backslash G(X)$ shows that $U$ satisfies PD.

Now we treat the general case. We write $G(X)=\left\{x_{1}, \ldots, x_{k}\right\}$. We choose for each $i$ an open neighborhood $V_{i}$ of $x_{i}$ such that $\left(V_{i}\right)_{i=1, \ldots, k}$ are pairwise disjoint. By intersecting with $U$ if necessary, we may assume that if $x_{i} \in U$, we have $V_{i} \subset U$. Let $J$ be the set of $i$ such that $x_{i} \in U$. Since $\bigcup_{i=1}^{k} V_{i}$ contains $G(X)$, it satisfies PD by our reduction above. Now by Lemma $4.6 \mathrm{ii}$ ) we know that $\bigcup_{j \in J} V_{j}$ also satisfies PD. We write $U_{1}=U \backslash G(X)$ and $U_{2}=\bigcup_{j \in J} V_{j}$. Both $U_{1}$ and $U_{12}$ satisfy PD since they do not contain any type II points of positive genus [JW18, Theorem 2]. Since we just showed that $U_{2}$ satisfies PD, so does $U$ by Lemma $4.6 i$ ).

4.3. Dimensions of cohomology and a conjecture by Y. Liu. For curves $X$ that satisfy $S_{X}=0$, Poincaré duality enables us to completely calculate the tropical Hodge numbers $h^{p, q}(U):=\operatorname{dim}_{\mathbb{R}} \mathrm{H}^{p, q}(U)$ both for $U=X^{\text {an }}$ as well as for a basis of the topology. The calculation of $h^{p, q}\left(X^{\text {an }}\right)$ enables us to establish the case of curves for a conjecture by Y. Liu.

For the case of a basis of the topology, we follow closely the proofs of Wanner and the author in [JW18, Section 5], using our stronger Poincaré duality results from Theorem 4.7 . 
Theorem 4.8. Let $X$ be a smooth projective curve over $K$ such that $S_{X}=0$. We denote by $b$ the dimension of the first singular cohomology $\mathrm{H}_{\text {sing }}^{1}\left(X^{\mathrm{an}}, \mathbb{R}\right)$ of $X^{\mathrm{an}}$. Then we have

$$
h^{p, q}\left(X^{\text {an }}\right)=\left\{\begin{array}{l}
1 \text { if }(p, q)=(0,0),(1,1) \\
b \text { if }(p, q)=(0,1),(1,0)
\end{array}\right.
$$

Note that $b$ is also the first Betti number of the incidence graph of a semistable model of $X$ or equivalently of any skeleton of $X^{\text {an }}$ [BPR13,4.9].

Proof. We have $h^{0,0}\left(X^{\text {an }}\right)=1$ and $h^{0,1}\left(X^{\text {an }}\right)=b$ by [Jel16b, Corollary 4.6]. Thus $h^{1,1}\left(X^{\text {an }}\right)=1$ and $h^{1,0}\left(X^{\text {an }}\right)=b$ follow from Theorem 4.2 .

For a variety $X$ over $K$ and $p \geq q$, Y. Liu constructed maps $N: \mathrm{H}^{p, q}\left(X^{\text {an }}\right) \rightarrow$ $\mathrm{H}^{q, p}\left(X^{\text {an }}\right)$ and conjectures that these are isomorphisms when $K$ is an algebraically closed non-archimedean field whose residue field is the algebraic closure of a finite field and $X$ is smooth and proper [Liu17a, Conjecture 5.2]. He established this for $(p, q)=(1,0)$ under some additional assumptions [Liu17a, Theorem 5.12]. We show that when $X$ is a curve, these additional assumptions are not necessary.

Theorem 4.9. Let $X$ be a smooth projective curve over $K$, where the residue field of $K$ is the algebraic closure of a finite field. Then Liu's conjecture holds for X.

Proof. The only case we have to consider is $(p, q)=(1,0)$. Then the map $N$ coincides with the operator $J$ [Liu17a, Lemma 3.2 (1)], which we know to be injective by [Jel16a, 3.4.11]. We have $S_{X}=0$ since the residue field of $K$ is the algebraic closure of a finite field. Thus by Theorem 4.8, the dimensions of $\mathrm{H}^{1,0}\left(X^{\text {an }}\right)$ and $\mathrm{H}^{0,1}\left(X^{\text {an }}\right)$ agree and are finite, which shows that the injective map $N$ is an isomorphism.

As in [JW18], for local considerations we use results on the structure of non-archimedean curves from [BPR13], as well as the notion of semistable vertex sets defined there. A semistable vertex set is a finite subset $V$ of $X^{\text {an }}$ such that $X^{\text {an }} \backslash V$ is isomorphic to a disjoint union of open balls and finitely many open annuli. The following definition is inspired by [BPR13, Corollary 4.27 \& Definition 4.28] and also appeared in [JW18, Definition 5.2].

Definition 4.10. Let $X$ be a smooth curve. An open subset $U$ of $X^{\text {an }}$ is called simple if it is either isomorphic to an open disc or an open annulus or if there exists a semistable vertex set $V$, with associated skeleton $\Sigma(X, V)$ and retraction $\tau_{V}$, and a simply connected open subset $W$ of $\Sigma(X, V)$ such that $U=\tau_{V}^{-1}(W)$. A simple open subset $U$ is called strictly simple if its closure in $X^{\text {an }}$ is simply connected in the case of a disc or an annulus or if the closure of $W$ in $\Sigma(X, V)$ is simply connected for $U=\tau_{V}^{-1}(W)$.

Remark 4.11. Strictly simple subsets form a basis of the topology and have finitely many boundary points [JW18, Proposition $5.3 \&$ Corollary 5.5]. If $U$ is a strictly simple open subset with $k$ boundary points, it is properly homotopy equivalent to the one point union of $k$ half open intervals, glued together at the closed ends [JW18, Lemma 5.6].

Theorem 4.12. Let $X$ be a smooth projective curve over $K$ such that $S_{X}=0$. Let $U$ be a strictly simple open subset of $X^{\text {an }}$ and denote by $k:=\# \partial U$ the finite number of boundary points. Then we have

$$
h^{p, q}(U)=\left\{\begin{array}{ll}
1 & \text { if }(p, q)=(0,0) \\
k-1 & \text { if }(p, q)=(1,0) \\
0 & \text { else }
\end{array} \text { and } h_{c}^{p, q}(U)= \begin{cases}1 & \text { if }(p, q)=(1,1) \\
k-1 & \text { if }(p, q)=(0,1) \\
0 & \text { else. }\end{cases}\right.
$$


Proof. First, note that $U$ has PD by Theorem 4.7. Since further $h^{p, q}(U)$ and $h_{c}^{p, q}(U)$ are zero unless $(p, q) \in\{0,1\}^{2}$, it is sufficient to calculate $h^{0, q}(U)$ and $h_{c}^{0, q}(U)$ for $q=0,1$. Since $\mathrm{H}^{0, q}(U) \cong \mathrm{H}_{\text {sing }}^{q}(U, \mathbb{R})$, we only have to calculate the Betti numbers $h_{\text {sing }}^{q}(U)$ and $h_{c, \text { sing }}^{q}(U)$ of singular cohomology, which are invariant under proper homotopy equivalences. Thus by Remark 4.11, we have to calculate $h_{\text {sing }}^{q}(Y)$ and $h_{c \text {,sing }}^{q}(Y)$ for $Y$ a one point union of $k$ intervals. The details are carried out in the proof of [JW18, Theorem 5.7].

\section{REFERENCES}

[Ber90] Vladimir G. Berkovich. Spectral theory and analytic geometry over non-Archimedean fields, volume 33 of Mathematical Surveys and Monographs. American Mathematical Society, Providence, RI, 1990.

[BPR13] Matthew Baker, Sam Payne, and Joseph Rabinoff. On the structure of non-Archimedean analytic curves. In Tropical and non-Archimedean geometry, volume 605 of Contemp. Math., pages 93-121. Amer. Math. Soc., Providence, RI, 2013.

[BT82] Raoul Bott and Loring W. Tu. Differential forms in algebraic topology, volume 82 of Graduate Texts in Mathematics. Springer-Verlag, New York-Berlin, 1982.

[CLD12] Antoine Chambert-Loir and Antoine Ducros. Formes différentielles réelles et courants sur les espaces de Berkovich. 2012. http://arxiv.org/abs/1204.6277.

[Gub16] Walter Gubler. Forms and currents on the analytification of an algebraic variety (after Chambert-Loir and Ducros). In Matthew Baker and Sam Payne, editors, Nonarchimedean and Tropical Geometry, Simons Symposia, pages 1-30, Switzerland, 2016. Springer.

[Jel16a] Philipp Jell. Differential forms on Berkovich analytic spaces and their cohomology. 2016. PhD Thesis, availible at http://epub.uni-regensburg.de/34788/1/Thesis Jell.pdf .

[Jel16b] Philipp Jell. A Poincaré lemma for real-valued differential forms on Berkovich spaces. Math. Z., 282(3-4):1149-1167, 2016.

[JSS15] Philipp Jell, Kristin Shaw, and Jascha Smacka. Superforms, tropical cohomology and Poincaré duality. 2015. http://arxiv.org/abs/1512.07409 to appear in Advances in Geometry.

[JW18] Philipp Jell and Veronika Wanner. Poincaré duality for the tropical Dolbeault cohomology of non-archimedean Mumford curves. J. Number Theory, 187:344-371, 2018.

[Liu17a] Yifeng Liu. Monodromy map for tropical Dolbeault cohomology. 2017. http://www.math.northwestern.edu/ liuyf/monodromy.pdf, to appear in Algebraic Geometry.

[Liu17b] Yifeng Liu. Tropical cycle classes for non-archimedean spaces and weight decomposition of de Rham cohomology sheaves. 2017. http://www.math.northwestern.edu/ liuyf/deRham.pdf.

[MZ08] Grigory Mikhalkin and Ilia Zharkov. Tropical curves, their Jacobians and theta functions. In Curves and abelian varieties, volume 465 of Contemp. Math., pages 203-230. Amer. Math. Soc., Providence, RI, 2008.

[Thu05] Amaury Thuillier. Théorie du potentiel sur les courbes en géométrie analytique non archimédienne. Applications à la théorie d'Arakelov. 2005.

[Wan16] Veronika Wanner. Harmonic functions on the Berkovich projective line. 2016. Master Thesis, availible at https://epub.uni-regensburg.de/35758/1/MA.pdf .

P. Jell, Georgia Institute of Technology, 686 Cherry Street, Atlanta, Ga 30332-0160

E-mail address: philipp.jell@math.gatech.edu 